\title{
Reducing the Motor Power Losses of a Four-Wheel Drive Fully Electric Vehicle via Wheel Torque Allocation
}

\author{
A. Pennycott, L. De Novellis, A. Sabbatini, P. Gruber and A. Sorniotti \\ Department of Mechanical Engineering Sciences \\ University of Surrey \\ United Kingdom
}

\section{Abstract}

Individually-controlled electric motors provide opportunities for enhancing the handling characteristics and energy efficiency of fully electric vehicles. Online power loss minimisation schemes based on electric motor efficiency data may, however, be impractical for real-time implementation due to the heavy computational demand. In this paper, the optimal wheel torque distribution for minimal power losses in the electric motor drives is evaluated in an offline optimisation procedure and then approximated using a simple function for online control allocation. The wheel torque allocation scheme is evaluated via a simulation approach incorporating constant speed driving at constant speed, ramp and step steer manoeuvres. The energy efficient wheel torque allocation scheme provides motor power loss reductions and yields savings in the total power utilisation compared to a simpler method in which the torques are evenly distributed across the four wheels. The method does not rely on complex online optimisation and can be applied on real electric vehicles in order to improve efficiency and thus reduce power consumption during different manoeuvres.

Keywords: fully electric vehicles; control allocation; energy efficiency

\section{Nomenclature}

\section{Symbols}

$\Delta \tau_{w} \quad$ Deviation of wheel torque from even torque allocation solution

$\delta_{w} \quad$ Wheel angle [rad]

$\gamma \quad$ Front to total wheel torque ratio for one vehicle side

$\gamma^{*} \quad$ Optimum front to total wheel torque ratio for one vehicle side

$\kappa \quad$ Transmission ratio

$\omega_{m} \quad$ Motor speed $[\mathrm{rad} / \mathrm{s}]$

$\tau_{m} \quad$ Motor torque $[\mathrm{Nm}]$

\footnotetext{
*a.pennycott@surrey.ac.uk
} 


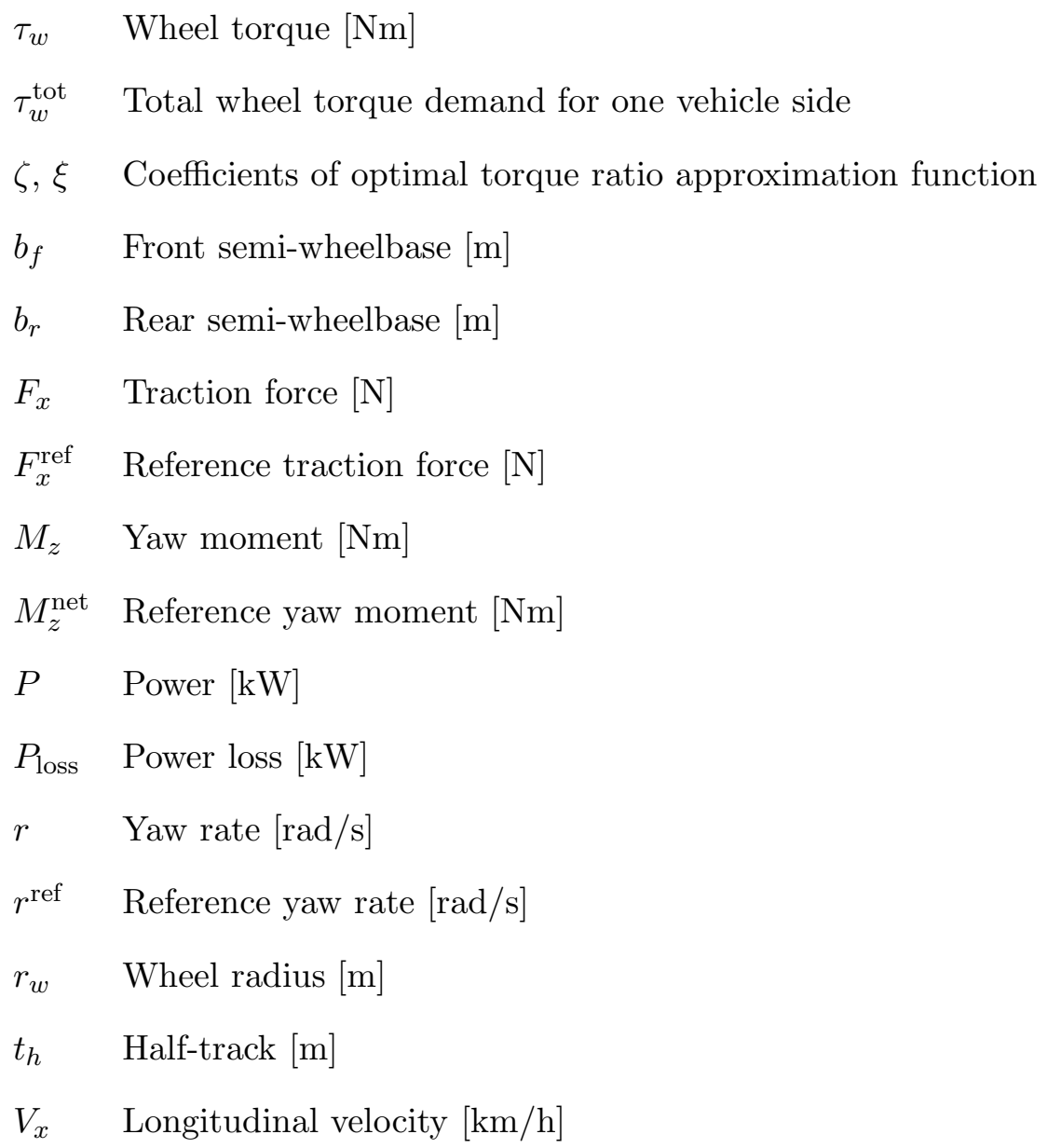

\section{Introduction}

Individually-controlled powertrains offer the possibility of enhancing the performance of fully electric vehicles in terms of their handling characteristics and fun-to-drive element. Continuously-operating control systems can alter the handling characteristics of a vehicle [1,2]. For instance, control systems can modify the understeer characteristic - the relationship between the steering wheel angle and lateral acceleration [3] - by making the car follow a target yaw rate based on the steering wheel angle, vehicle velocity and longitudinal acceleration [4]. This fundamentally changes the manner in which the vehicle responds to steering wheel inputs from the driver.

In addition to realising fundamental vehicle dynamics modifications, the use of four electric motors that can be individually controlled allows secondary considerations such as power minimisation and tyre slip reduction to be included during control allocation (in which the actuation levels for the individual motors are assigned). For example, a high level controller could produce traction force and yaw moment targets which can be realised via an infinite number of combinations of the four individual motor torques. The targets could be met using only the actuators of the wheels at the front axle, or alternatively, using even contributions from the wheels of the front and rear axles. These combinations will differ in terms of the total energy consumption and longitudinal tyre slip. A function relating such quantities with the wheel torques produced by the control allocation algorithm thus allows secondary goals to be satisfied as well as realising the primary aim of vehicle dynamics modification.

Indeed, there are a variety of cost functions for control allocation optimisation which relate tyre slip [5], actuator effort [6] and power losses from the electric motor drives [7, 8, 9] with the torques 
produced at the four wheels. The latter studies demonstrate a marginal power saving using an optimisation procedure during wheel torque allocation. The utility of control allocation in reducing power losses from the electric motor drives has also been demonstrated in an investigation using an offline optimisation procedure in which power loss reductions were achieved for three different motor types [10]. However, while the latter study determined the potential for power loss reductions, the method could not be directly applied in online applications in real-time due to the computationallyintensive minimisation procedures used.

Direct optimisation based on efficiency data online may, however, be challenging. For fourwheel drive vehicles where each of the four wheels is fitted with the same motor type, quadratic programming - a form of optimisation that can be practically implemented online using the active set method, for example $[11,12]$ - is unlikely to yield significant energy savings due to the symmetry of the second order power loss cost function [10]. Online minimisation of a higher order polynomial (e.g. used in [7]), in addition to the challenges posed by the existence of multiple local minima, could be impractical for actual implementation on real vehicles due to the high computational demands involved with minimising a complex cost function.

In this study, the control allocation problem is simplified and an offline procedure is applied to the reduced formulation in order to determine wheel torques that minimise the motor power losses, based on motor efficiency data from the manufacturer. The focus is on generating a simple method that can be implemented practically on real vehicles. A function is used to approximate the results of the offline optimisation for online wheel torque allocation. Using a simulation approach, the power losses incurred by the novel control allocation scheme during straight-ahead, ramp and step steer manoeuvres are compared to those produced by a simpler allocation scheme in which the torques are allocated evenly from the actuators of the front and rear axles.

\section{Methods}

\subsection{High Level Control and Driving Modes}

The handling characteristics of the studied vehicle are changed by imposing a reference yaw rate, $r^{\text {ref }}$. For each combination of steering wheel angle, vehicle speed, and longitudinal acceleration, the reference yaw rate is calculated based on a look-up table, formulated by means of an offline optimisation procedure [4]. Tracking the resulting target yaw rate allows the vehicle to achieve a desirable understeer characteristic.

The combination of the high level controller and control allocation thus enables different target understeer characteristics to be realised, corresponding to different 'driving modes'. For instance, when the 'sport' driving mode used in this study is active, the car reacts quickly to changes in the steering wheel angle. The sport driving mode is realised by setting a low understeer gradient. Moreover, for a passive (uncontrolled) vehicle, the understeer characteristic typically varies with the longitudinal acceleration; this variation can be eliminated using continuous control, giving a constant understeer gradient for all longitudinal accelerations. Finally, the maximum lateral acceleration achievable can be extended through the continuously-acting controller. The understeer characteristics of the passive vehicle and the car under the sport driving mode are shown in figure 1. This figure highlights the impact of the control scheme on the handling characteristics.

The actual yaw rate, $r$, is driven to the target, $r^{\text {ref }}$, through a corrective yaw moment, $M_{z}^{\text {ref }}$, determined by a high level controller with feedforward and proportional-integral-derivative elements described in [4]. The control allocation scheme then assigns wheel torques such that these demands are realised, and appropriate motor torques and brake pressures are then applied to deliver the required wheel torques. 


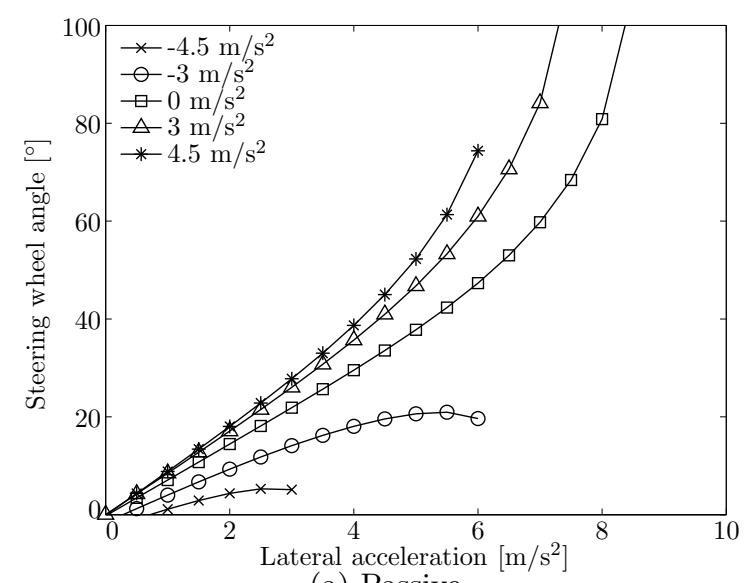

(a) Passive

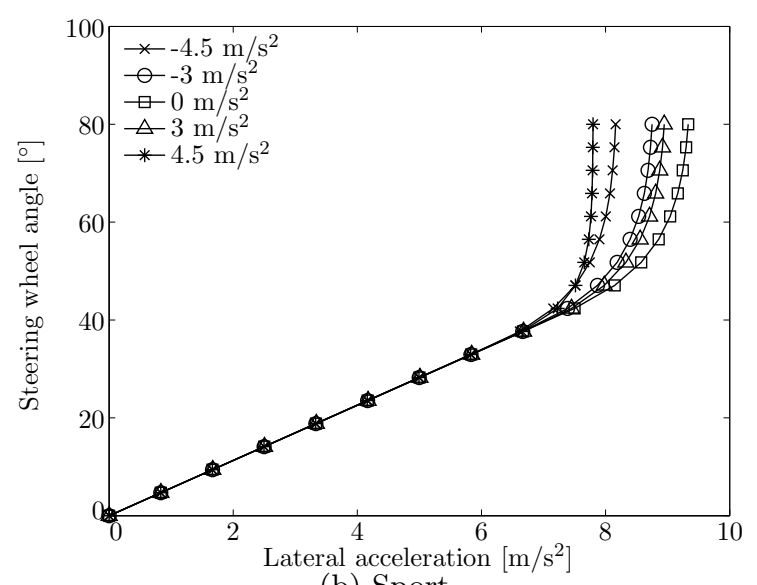

(b) Sport

Figure 1: Understeer characteristics at different longitudinal accelerations for the passive vehicle and sport driving mode. The main objective of the control allocation scheme is to realise the sport understeer characteristic through assigning wheel torques such that a target yaw moment is produced.

\subsection{Energy Efficient Wheel Torque Allocation}

\subsubsection{Simplified Control Allocation}

The basic vehicle geometry is shown in figure 2. The front-left, front-right, rear-left and rear-right wheels are respectively given the subscripts 1, 2, 3 and 4 throughout this paper. Using the half track, $t_{h}$, front and rear semi-wheelbases, $b_{f}$ and $b_{r}$, and the wheel radius, $r_{w}$, the four wheel torques, $\tau_{w, 1-4}$, can be related to the net traction force, $F_{x}$, and yaw moment, $M_{z}$, acting on the vehicle.

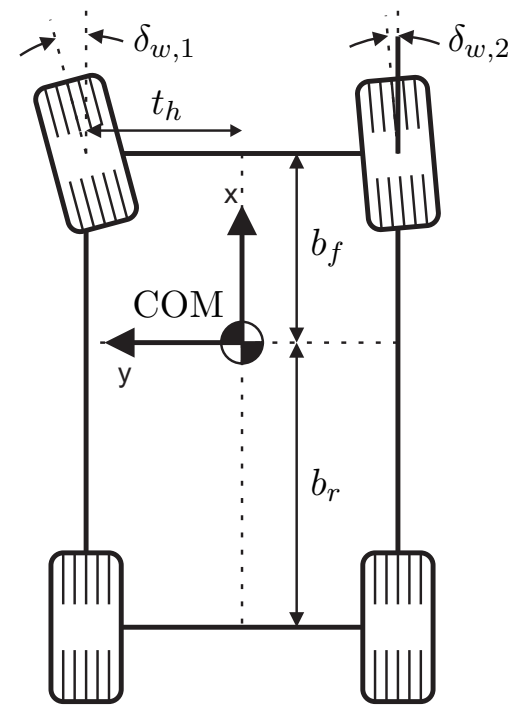

Figure 2: Vehicle geometry used for control allocation. 


$$
\begin{gathered}
F_{x}=\frac{1}{r_{w}}\left(\tau_{w, 1} \cos \delta_{w, 1}+\tau_{w, 2} \cos \delta_{w, 2}+\tau_{w, 3}+\tau_{w, 4}\right) \\
M_{z}=\frac{1}{r_{w}}\left(\left(-t_{h} \cos \delta_{w, 1}+b_{f} \sin \delta_{w, 1}\right) \tau_{w, 1}+\left(t_{h} \cos \delta_{w, 2}+b_{f} \sin \delta_{w, 2}\right) \tau_{w, 2}-t_{h} \tau_{w, 3}+t_{h} \tau_{w, 4}\right)
\end{gathered}
$$

Assuming small wheel angles, the two equations become

$$
\begin{gathered}
F_{x}=\frac{1}{r_{w}}\left(\tau_{w, 1}+\tau_{w, 2}+\tau_{w, 3}+\tau_{w, 4}\right) \\
M_{z}=\frac{t_{h}}{r_{w}}\left(-\tau_{w, 1}+\tau_{w, 2}-\tau_{w, 3}+\tau_{w, 4}\right) .
\end{gathered}
$$

Using the null space of linear equations (3) and (4), the general solution for the wheel torque vector, $\boldsymbol{\tau}_{w}$, can be expressed as a function of two independent variables, $\Delta \tau_{w, l}$ and $\Delta \tau_{w, r}$. The latter variables represent wheel torque deviations from the solution corresponding to even contributions from the front and rear wheels to the traction force and yaw moment targets.

$$
\boldsymbol{\tau}_{w} \triangleq\left[\begin{array}{c}
\tau_{w, 1} \\
\tau_{w, 2} \\
\tau_{w, 3} \\
\tau_{w, 4}
\end{array}\right]=\frac{r_{w}}{4}\left[\begin{array}{l}
F_{x}-M_{z} / t_{h} \\
F_{x}+M_{z} / t_{h} \\
F_{x}-M_{z} / t_{h} \\
F_{x}+M_{z} / t_{h}
\end{array}\right]+\left[\begin{array}{r}
-\Delta \tau_{w, l} \\
-\Delta \tau_{w, r} \\
\Delta \tau_{w, l} \\
\Delta \tau_{w, r}
\end{array}\right] .
$$

The sum of torques on the left-hand side is thus constant, being equal to $\frac{r_{w}}{2}\left(F_{x}-M_{z} / t_{h}\right)$. Analogously, the sum of torques on the right-hand wheels is also constant, and equal to $\frac{r_{w}}{2}\left(F_{x}+M_{z} / t_{h}\right)$. Wheel torque allocation for the left-hand and right-hand wheels can thus be approached separately. For both sides of the car, the problem consists of determining the proportions of the torque demand met by the wheels of the front and rear axles.

Let $\gamma_{l}$ and $\gamma_{r}$ be defined as follows:

$$
\begin{aligned}
\gamma_{l} & =\frac{\tau_{w, 1}}{\tau_{w, 1}+\tau_{w, 3}} \\
\gamma_{r} & =\frac{\tau_{w, 2}}{\tau_{w, 2}+\tau_{w, 4}} .
\end{aligned}
$$

$\gamma_{l}$ and $\gamma_{r}$ thus represent the ratio of the front-left torque to total torque on the left-hand side of the car, and the ratio of the front-right torque to total torque on the right-hand side of the car, respectively.

\subsubsection{Offline Optimisation}

Different values of $\gamma_{l}$ and $\gamma_{r}$ cause the motor units to operate in different regions of the torque-speed space where the overall efficiency and power losses vary. In general, the values of $\gamma_{l}$ and $\gamma_{r}$ at which the power losses are minimised depend on the motor speed and the total torque demands on the left- and right-hand sides of the vehicle. Let $\gamma_{l}^{*}$ and $\gamma_{r}^{*}$ denote the minimising values of the ratios.

In order to determine the optimal front-to-total torque ratios, a function which evaluates the power losses at different levels of $\gamma_{l}$ and $\gamma_{r}$, torque demand and motor speed is needed. Let $\tau_{w}^{\text {tot }}$ be the total wheel torque demand for one side of the vehicle (either left or right). Using the motor maps provided by the manufacturer, the efficiency of motor $i, \eta_{i}$, at motor torque $\tau_{m, i}$ and motor speed $\omega_{m, i}$ can be determined, allowing the computation of the motor power dissipation at each actuator.

$$
P_{\mathrm{loss}, i}= \begin{cases}P_{i}\left(\frac{1}{\eta_{i}}-1\right) & \text { if } P_{i}>=0 \\ P_{i}\left(1-\eta_{i}\right) & \text { if } P_{i}<0\end{cases}
$$


In this expression, the power output, $P_{i}$, is given by the product of motor torque and speed.

It may be assumed that the wheel speeds at the front-left and rear-left wheels are approximately equal, with an analogous assumption for the right-hand wheels. For one side of the vehicle, the front and rear wheel torques, $\tau_{w, f}$ and $\tau_{w, r}$, are

$$
\begin{aligned}
\tau_{w, f} & =\gamma \tau_{w}^{\mathrm{tot}} \\
\tau_{w, r} & =(1-\gamma) \tau_{w}^{\mathrm{tot}}
\end{aligned}
$$

and thus the total power loss from the actuators at the given vehicle side is

$$
P_{\text {loss }}\left(\gamma, \omega_{m}, \tau_{w}^{\text {tot }}\right)=P_{\text {loss }, f}\left(\gamma, \omega_{m}, \tau_{w}^{\text {tot }}\right)+P_{\text {loss }, r}\left(\gamma, \omega_{m}, \tau_{w}^{\text {tot }}\right) .
$$

At each operating point in terms of $\omega_{m}$ and $\tau_{w}^{\text {tot }}$ (i.e. the motor speed and total torque demand), a cost function $f$ can be written in the front-to-total wheel torque ratio:

$$
f\left(\gamma, \omega_{m}, \tau_{w}^{\mathrm{tot}}\right)=P_{\mathrm{loss}}\left(\gamma, \omega_{m}, \tau_{w}^{\mathrm{tot}}\right) .
$$

In addition to minimising the power loss at $\omega_{m}$ and $\tau_{w}^{\text {tot }}$, the optimisation scheme takes into account the maximum and minimum motor torques, $\tau_{m}^{\max }$ and $\tau_{m}^{\min }$, that can be produced at the specified motor speed. Using the transmission ratio, $\kappa$, the wheel and motor torques are approximately related in steady state as $\tau_{w}=\kappa \tau_{m}$. Furthermore, to avoid oversteering behaviour, wheel torques predominantly from the front axle are favoured so that $\gamma$ should be greater than 0.5 . This leads to the equations

$$
\begin{gathered}
\tau_{m}^{\min } \leq \frac{1}{\kappa} \gamma \tau_{w}^{\text {tot }} \leq \tau_{m}^{\max } \\
\tau_{m}^{\min } \leq \frac{1}{\kappa}(1-\gamma) \tau_{w}^{\text {tot }} \leq \tau_{m}^{\max } \\
0.5 \leq \gamma \leq 1 .
\end{gathered}
$$

These constraints can be transformed into standard form for the optimisation scheme using the variable $h$. For $\tau_{w}^{\text {tot }} \geq 0$ :

$$
\begin{aligned}
& h_{1}\left(\gamma, \omega_{m}, \tau_{w}^{\mathrm{tot}}\right)=\gamma-\max \left(0.5, \frac{\kappa \tau_{m}^{\min }}{\tau_{w}^{\mathrm{tot}}}, 1-\frac{\kappa \tau_{m}^{\max }}{\tau_{m}^{\mathrm{tot}}}\right) \geq 0 \\
& h_{2}\left(\gamma, \omega_{m}, \tau_{w}^{\mathrm{tot}}\right)=-\gamma+\min \left(1, \frac{\kappa \tau_{m}^{\max }}{\tau_{w}^{\mathrm{tot}}}, 1-\frac{\kappa \tau_{m}^{\min }}{\tau_{w}^{\text {tot }}}\right) \geq 0 .
\end{aligned}
$$

Analogous constraints can be defined in this form for $\tau_{w}^{\text {tot }}<0$.

The optimisation problem can now be expressed in standard form:

$$
\min _{\gamma} f\left(\gamma, \omega_{m}, \tau_{w}^{\text {tot }}\right)
$$

subject to

$$
\begin{aligned}
& h_{1}\left(\gamma, \omega_{m}, \tau_{w}^{\mathrm{tot}}\right) \geq 0 \\
& h_{2}\left(\gamma, \omega_{m}, \tau_{w}^{\mathrm{tot}}\right) \geq 0 .
\end{aligned}
$$

Vectors of motor speed and torque demand are generated (with elements $\omega_{m, i}$ and $\tau_{w, j}^{\text {tot }}$ ) in order to produce a grid of motor speed and torque values. At each grid point (with indices $i$ and $j$ ), the corresponding optimal wheel torque, $\gamma_{i, j}^{*}$, is calculated using the interior point method. Various starting points for the optimisation are used for each grid point to give a number of local minima for each condition, with the global minimum point being selected from the set of local minima. 


\subsubsection{Approximation Function for Online Application}

Using parameter $\zeta$, a simple function is used to approximate $\gamma^{*}$ throughout the torque-speed grid structure via the function $\hat{\gamma}^{*}$.

$$
\gamma_{i, j}^{*} \approx \zeta_{2, i}+0.5\left(\zeta_{3, i}-\zeta_{2, i}\right) \tanh \left(\zeta_{4, i}\left(\tau_{w, j}^{\mathrm{tot}}-\zeta_{1, i}\right)\right)=\hat{\gamma}^{*}\left(\tau_{w, j}^{\mathrm{tot}}, \zeta_{1, i}, \zeta_{2, i}, \zeta_{3, i}, \zeta_{4, i}\right)
$$

The parameters $\zeta_{1-4, i}$ depend on the corresponding motor speed, $\omega_{m, i}$. The shape of this function for different values of total torque demand was chosen based on initial optimisation results, and is illustrated in figure 3 .

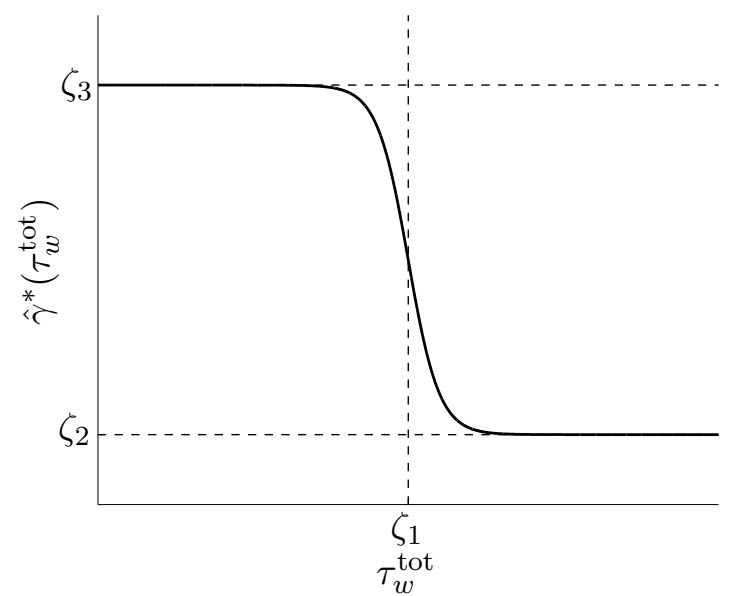

Figure 3: Form of the online approximation function at constant motor speed for online optimal wheel torque allocation.

At each level of motor speed in the grid structure, the coefficients are determined from the offline optimisation data via a least squares approach. The parameters of the problem are solved via the minimisation:

$$
\min _{\zeta_{1, i}, \zeta_{2, i}, \zeta_{3, i}, \zeta_{4, i}} \sum_{j}\left(\gamma_{i, j}^{*}-\hat{\gamma}^{*}\left(\tau_{w, j}^{\mathrm{tot}}, \zeta_{1, i}, \zeta_{2, i}, \zeta_{3, i}, \zeta_{4, i}\right)\right)^{2}
$$

For ease of online application, a fifth order polynomial with coefficients contained in matrix $\boldsymbol{\xi}$ is used to approximate the relationship between each coefficient $\zeta$ and the corresponding motor speed.

$$
\zeta_{k, i} \approx \sum_{l=0}^{5} \xi_{k, l}\left(\omega_{m, i}\right)^{l}
$$

This allows the optimal wheel torque at an arbitrary motor speed and torque demand to be expressed as a function, $g$, of the parameter matrix $\boldsymbol{\xi}$.

$$
\gamma^{*}\left(\omega_{m}, \tau_{w}^{\mathrm{tot}}\right) \approx g\left(\omega_{m}, \tau_{w}^{\mathrm{tot}}, \boldsymbol{\xi}\right)
$$

Two separate functions of this form - approximating the optimisation results for positive and negative wheel torque totals - are determined from the offline optimisation results through a least squares approach.

\subsubsection{Online Energy Efficient Wheel Torque Allocation}

Figure 4 shows how the approximation function is used online to determine the four wheel torques. Firstly, the wheel torque totals at the left- and right-hand sides of the vehicle, $\tau_{w, l}^{\text {tot }}$ and $\tau_{w, r}^{\text {tot }}$, are 
determined from equations (3) and (4). Next, the approximation function yields the (approximate) optimal front-to-total torque ratios, $\gamma_{l}^{*}$ and $\gamma_{r}^{*}$. The traction force and yaw moment targets are used in conjunction with the torque ratios in equations (1), (2), (6) and (7) in order to evaluate the four wheel torques.

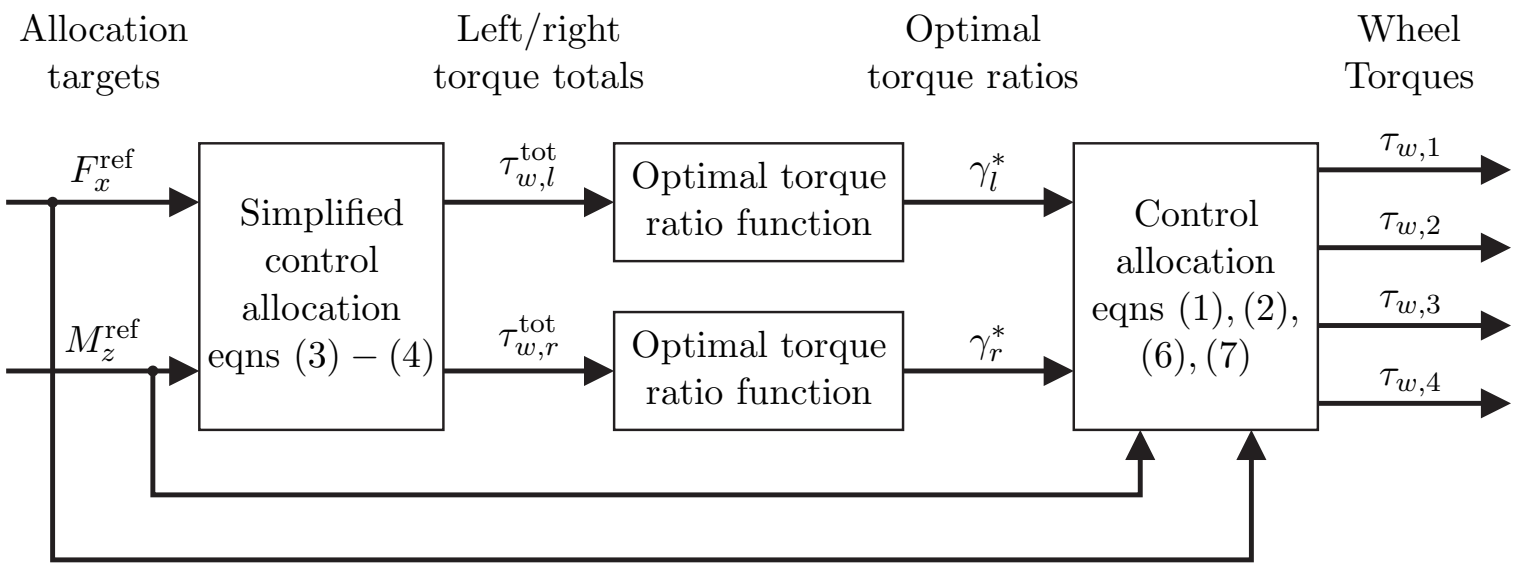

Figure 4: Schematic for online energy efficient control allocation scheme.

\subsection{Evenly Distributed Wheel Torque Allocation}

The energy efficient scheme is compared with a simpler method in which the wheel torques at the front and rear axles contribute equally to the traction force and yaw moment targets of the high level controller. Using approximations for the traction force and yaw moment equations (equations (1) and (2)), if the contribution of the front axle wheels to the total traction force demand is to be equal to that of the rear axle, the following equation applies.

$$
\frac{\tau_{w, 1}+\tau_{w, 2}}{\tau_{w, 1}+\tau_{w, 2}+\tau_{w, 3}+\tau_{w, 4}}=0.5
$$

Similarly, if the wheels at the front axle and those of the rear axle must create an equal yaw moment,

$$
\frac{-\tau_{w, 1}+\tau_{w, 2}}{-\tau_{w, 1}+\tau_{w, 2}-\tau_{w, 3}+\tau_{w, 4}}=0.5 .
$$

The set of equations (1), (2), (25) and (26) are solved in order to determine the required four wheel torques.

\subsection{Electric Motor Characteristics}

The motor used in this investigation is a switched reluctance motor. Data concerning efficiency throughout the motor torque-speed space is available in map form from the manufacturer; a contour plot of the motor efficiency is given in figure 5 .

\subsection{Vehicle Characteristics}

The vehicle is a four-wheel drive sport utility vehicle of mass $1963 \mathrm{~kg}$, half-track $0.81 \mathrm{~m}$ and front and rear semi-wheelbases of $1.1 \mathrm{~m}$ and $1.6 \mathrm{~m}$. The wheel radius is $0.36 \mathrm{~m}$ and the centre of mass is located $0.66 \mathrm{~m}$ above the ground. The car has four individually-controlled electric motors, with each powertrain having a fixed transmission ratio of 10:1. 


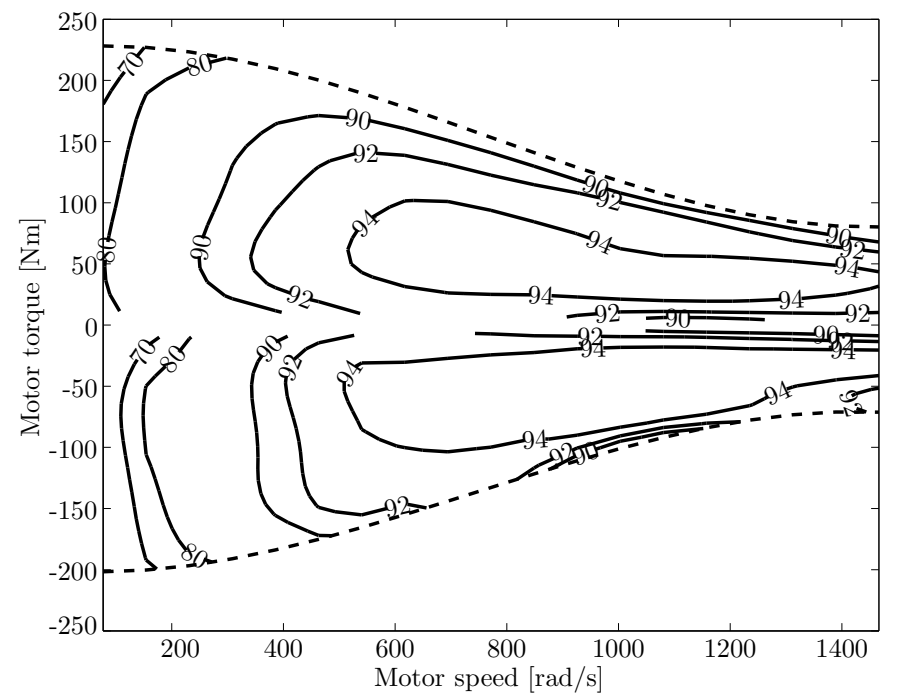

Figure 5: Efficiency contours (\%) of the motor at different levels of torque and speed.

\subsection{Simulation Study}

An experimentally-validated vehicle model developed in IPG CarMaker is simulated in this study. The model incorporates a powertrain model including the torsional dynamics of the half-shafts and the transmission backlash. Examples of validation data for a ramp steer manoeuvre and a sequence of step steers are shown in figure 6 . It can be seen that the yaw rate data predicted by the IPG CarMaker simulations are closely matched with experimental data.

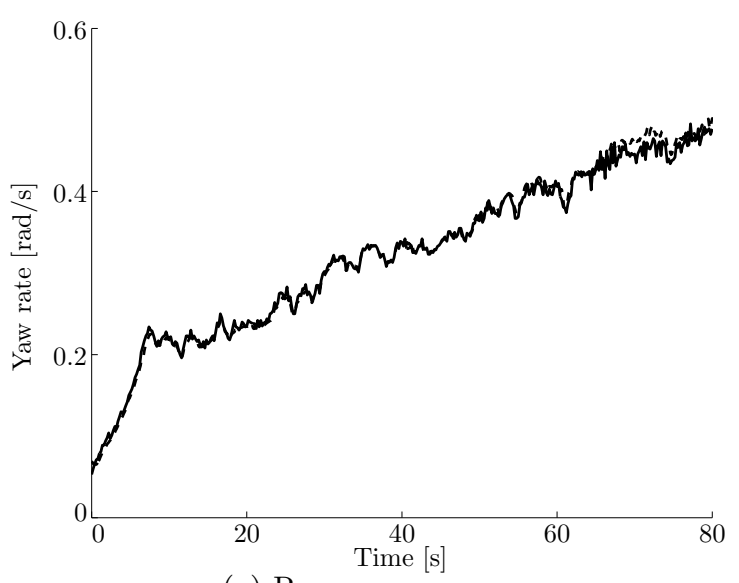

(a) Ramp manoeuvre

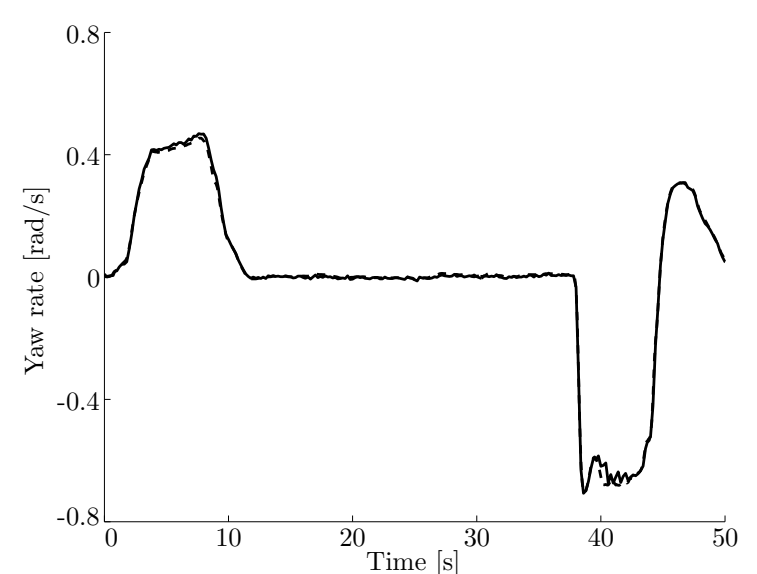

(b) Steps manoeuvre

Figure 6: Model validation data. Dashed and solid lines represent simulation and experimental data, respectively.

Three different manoeuvre types are investigated. The first is straight-ahead driving at constant speed. Manoeuvres at $50 \mathrm{~km} / \mathrm{h}$ and $100 \mathrm{~km} / \mathrm{h}$ are simulated. Secondly, there is a ramp steer manoeuvre with a constant steering wheel rate of $10^{\circ} / \mathrm{s}$ and longitudinal velocity of $100 \mathrm{~km} / \mathrm{h}$. The third manoeuvre is a sequence of step steers of different amplitudes, again at a constant velocity of $100 \mathrm{~km} / \mathrm{h}$. The motor power losses incurred during the ramp and step steer sequence manoeuvres under the energy efficient wheel torque distribution method (section 3.2) are compared with the 
losses incurred by the scheme in which the wheel torques are allocated evenly across the front and rear axles (section 3.3).

\section{Results}

\subsection{Offline Optimisation and Approximation}

Contour plots of $\gamma^{*}$, the ratio of the front to total wheel torque that yields minimal motor power loss, are given in figure 7. The left-hand plot shows the original values of $\gamma^{*}$ as given by the offline optimisation procedure and the right-hand plot shows the values of $\gamma^{*}$ as given by the approximation of equation (21) used in the online wheel torque allocation scheme. The normalised root mean square error between the offline optimisation solutions and the values from the approximation function is $7 \%$.

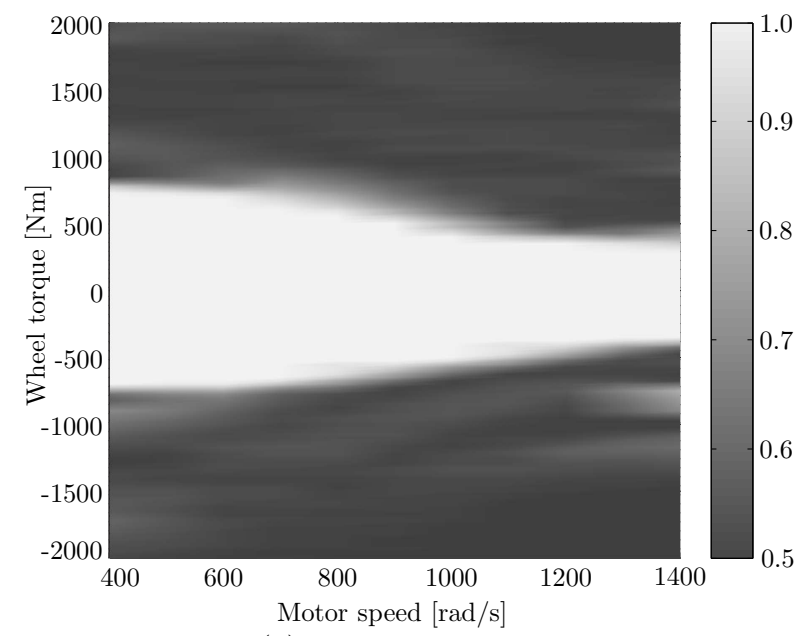

(a) Optimal solutions

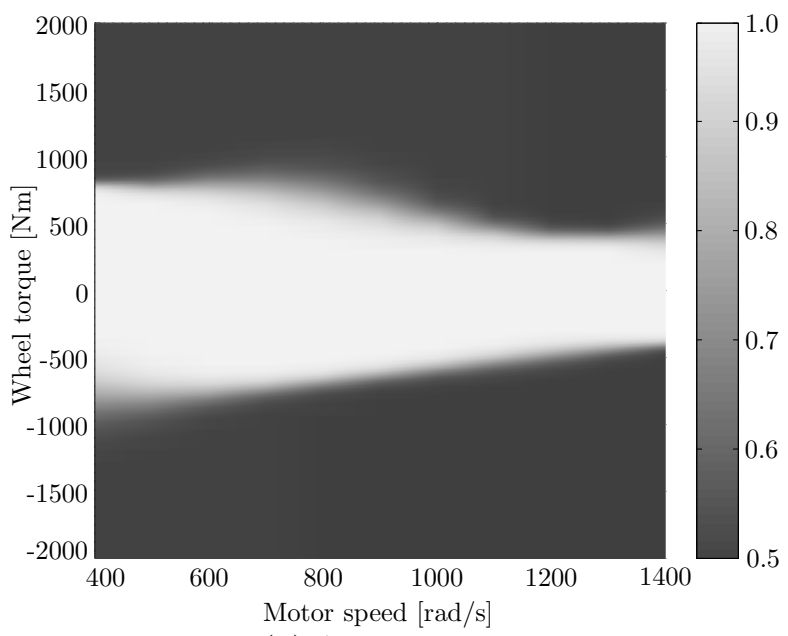

(b) Approximation

Figure 7: Optimal levels of the front-to-total wheel torque ratio as determined by the offline optimisation procedure. The optimal solutions and function approximation of equation (21) are shown.

The optimal levels of the front-to-total torque ratio follow a striking pattern. At a given motor speed and at low levels of wheel torque demand, a wheel torque allocation realising the entire torque demand for each side of the car from the front axle only is more efficient. As the total torque demand increases, there is a sharp decrease in $\gamma^{*}$ to around 0.5 , indicating that wheel torque allocation in which the front and rear axle wheels equally share the torque demand is more efficient.

\subsection{Online Wheel Torque Allocation}

The yaw rate responses of the vehicle during the ramp and multiple step steers manoeuvre are shown in figure 8. The wheel torque allocation enables accurate tracking of the reference yaw rate in each case by realising a corrective yaw moment set by the high level controller.

During both straight-ahead driving tests (at 50 and $100 \mathrm{~km} / \mathrm{h}$ ), $\gamma_{l}^{*}$ and $\gamma_{l}^{*}$ assume a constant value of 1 . Figure 9 illustrates the variation of the front-to-total torque ratios at the left- and righthand sides of the car $\left(\gamma_{l}^{*}\right.$ and $\gamma_{r}^{*}$, respectively) throughout the ramp and step manoeuvres. For the ramp steer manoeuvre (a left turn), the torque demands of the right-hand side of the car are higher. Consequently, while it is initially more efficient to derive all the wheel torque from the front axle 


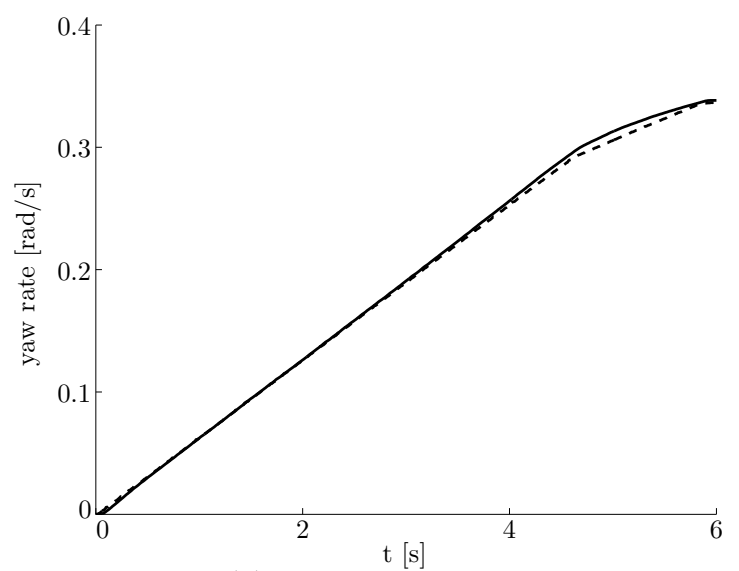

(a) Ramp manoeuvre

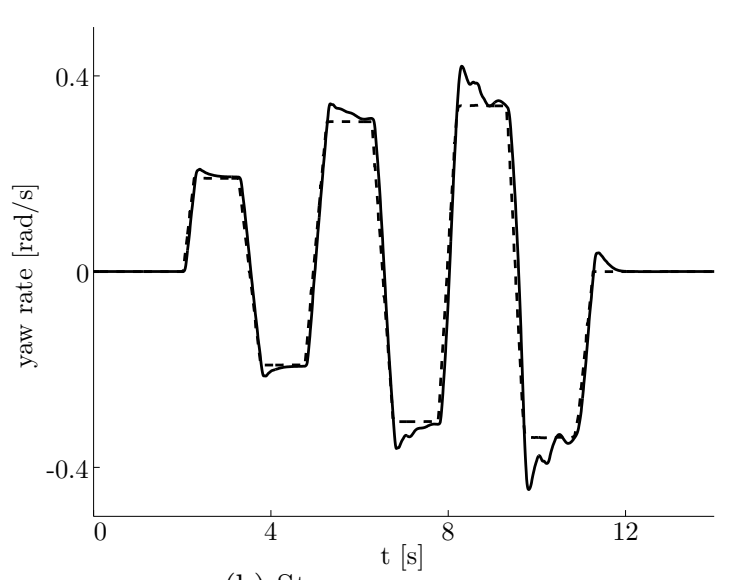

(b) Steps manoeuvre

Figure 8: Yaw rate response during ramp and step steers manoeuvres under the efficient wheel torque allocation scheme.

$\left(\gamma_{r}^{*}=1\right)$, after a couple of seconds, the most efficient right-hand side wheel torque distribution is an equal contribution from the front and rear wheels $\left(\gamma_{r}^{*}=0.5\right)$ due to the higher torque demand from the right-hand wheels $\left(\tau_{w, r}^{\text {tot }}\right)$.

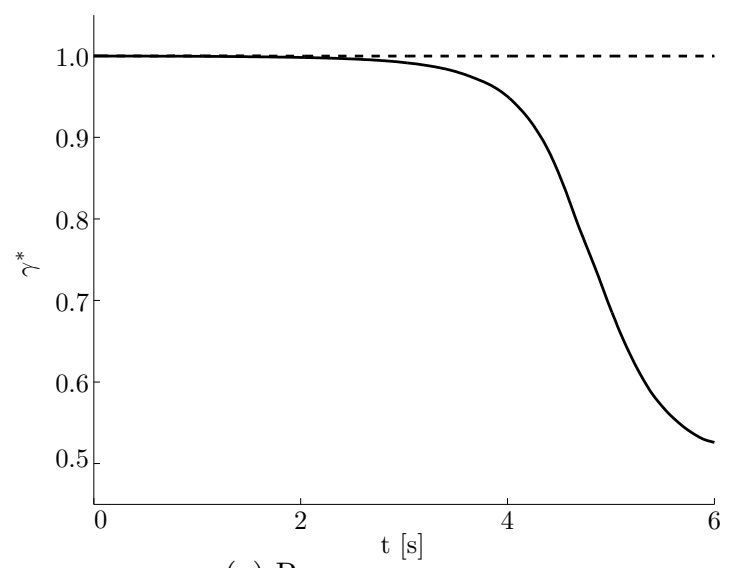

(a) Ramp manoeuvre

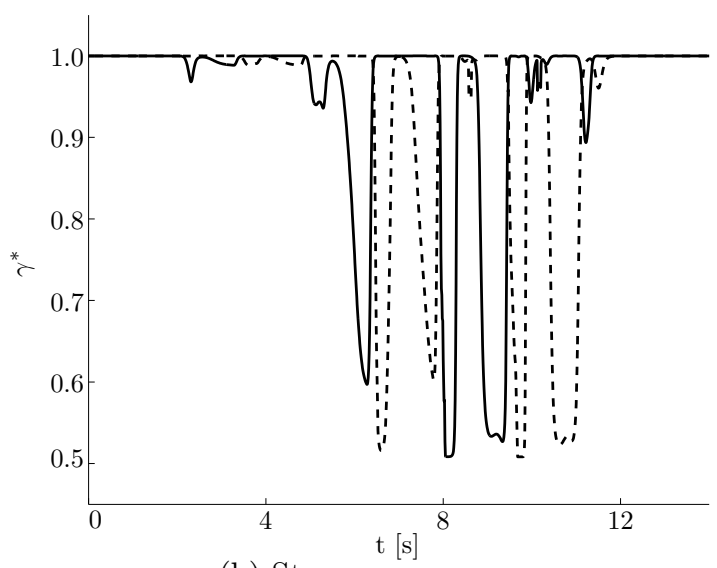

(b) Steps manoeuvre

Figure 9: Front-to-total torque ratios $\left(\gamma^{*}\right)$ during the ramp and step steers manoeuvres. The dashed and solid lines represent the ratios at the left- and right-hand sides of the vehicle $\left(\gamma_{l}^{*}\right.$ and $\left.\gamma_{r}^{*}\right)$.

The mean motor power losses incurred during the different manoeuvres under the two allocation schemes are shown in table 1 . Though the energy efficient scheme has little impact during the straight ahead manoeuvre at $50 \mathrm{~km} / \mathrm{h}$, at $100 \mathrm{~km} / \mathrm{h}$, the motor power losses are $3.1 \%$ lower when the optimisation-based scheme is applied. Furthermore, the motor power losses when the optimal allocation scheme is used are lower than those induced by the even distribution approach by $5.8 \%$ and $4.5 \%$ for the ramp steer and sequence of step steers manoeuvres, respectively. 


\begin{tabular}{|l|c|c|}
\hline Manoeuvre & Even allocation & Efficient allocation \\
\hline Straight-ahead at $50 \mathrm{~km} / \mathrm{h}$ & $0.519 \mathrm{~kW}$ & $0.518 \mathrm{~kW}$ \\
\hline Straight-ahead at $100 \mathrm{~km} / \mathrm{h}$ & $1.28 \mathrm{~kW}$ & $1.24 \mathrm{~kW}$ \\
\hline Ramp & $2.59 \mathrm{~kW}$ & $2.44 \mathrm{~kW}$ \\
\hline Steps & $2.92 \mathrm{~kW}$ & $2.79 \mathrm{~kW}$ \\
\hline
\end{tabular}

Table 1: Mean motor power losses for tested manoeuvres under even and efficient wheel torque allocation schemes.

\section{Discussion}

A method employing a simplication of the underlying wheel torque allocation problem in combination with an offline minimisation procedure has been proposed to reduce the power losses from the electric motor drives during various manoeuvres of a four-wheel drive fully electric vehicle. The more complex map evaluating the minimising levels of front-to-total wheel torque allocation at different motor speeds and total torque demands is represented via a simple function that can easily be evaluated online with little computational effort, in contrast with alternative approaches based on optimisation. The approach achieves motor power loss savings of up to around $6 \%$ and small reductions in overall power utilisation compared with even allocation of the traction force and yaw moment demands to the front and rear axles.

The transition between even wheel torque distribution and front-axle only allocation causes fluctuations in the assigned wheel torques which may give rise to drivability issues. However, this variation should not lead to significant comfort issues: the standard deviation of the car pitch angle is greater for the energy efficient method than the even distribution scheme by only $1 \%$ for the ramp and step steers manoeuvres.

The offline optimisation results show that for lower torque demands, it is more efficient to use only one motor (per vehicle side) to meet the wheel torque demand. This is in agreement with other research e.g. [9] which sought to improve energy efficiency by splitting the torque demands between front and rear actuators. For motors with more a pronounced variation of efficiency throughout the torque-speed space, it is likely that even greater power loss savings could be made. It is, however, feasible that other patterns of optimal torque distribution could arise for motors with different efficiency characteristics to the motor of this study. The offline optimisation could be used without modification for these cases, and a more complex function such as a neural network [13] or a look-up table [14] could be used for online approximation of the optimisation results for real time wheel torque allocation.

During other types of manoeuvres involving a significant braking effort, wheel torque allocation may have to operate within additional constraints, such as the braking regulations of the Economic Commission for Europe [15]. Furthermore, other limitations may arise from the maximum power that can be drawn from the battery. In order to meet these constraints while simultaneously satisfying traction force and yaw moment targets and also delivering the required front-to-total wheel torque distribution for minimal power losses, the allocation scheme could be formulated as an optimisation task. The traction force and yaw moment targets can be related to the wheel torques using the standard virtual control vector and control effectiveness matrix approach [16], and the required wheel torque ratio can be transformed into a quadratic cost function to be solved using the active set or interior point methods [17], among others.

This investigation has focused only on power losses from the electric motor drives. It should be noted that there are several sources of power loss during the operation of a fully electric vehicle: losses due to tyre slip, aerodynamic drag, transmission losses and dissipations from the battery. 
Some of these - particularly power dissipations due to tyre slip - are strongly influenced by control allocation. As a result, a reduction in the motor power losses may have only a limited impact on the overall power utilisation. For instance, although the motor power losses are reduced (compared with even torque allocation) by more than $5 \%$ when the optimal wheel torque allocation is applied during the sequence of step steers, the motor power losses account for only $6 \%$ of the total power loss for this manoeuvre. As a result, the reduction in the total power loss (and hence utlisation) is less than 1\%. Future control allocation algorithms should take into account different sources of dissipation, including power losses both due to tyre slip and from the electric motor drives in order to have a greater impact on the total power utilisation.

In addition to optimising a secondary cost function as is the focus of this paper, control allocation is frequently used to deal with situations of actuator failure. Very little modification of the control allocation scheme presented here is required to cope with situations in which one of the electric motors malfunctions. In this scenario, the corresponding front-to-total wheel torque ratios would be set to 0 or 1 (for failure of the front or rear actuators, respectively). Terms corresponding to the malfunctioning actuator would then be removed from equations (1) and (2) and the resulting linear equations solved to give the appropriate wheel torques as before.

\section{Conclusions}

A method for reducing power losses from the electric motor drives for online wheel torque allocation of fully electric vehicles has been proposed which utilises an offline minimisation procedure based on motor efficiency data and a simple function to approximate the optimal solutions for online application. The offline optimisation shows that for minimum power losses at low torque demands, the left- and right-hand wheel torque demands should be realised using only the motors of the front axle; higher wheel torque targets should be delivered equally by the front and rear motors at each side of the vehicle. The online method provides lower motor power losses and overall power utilisation during straight-ahead, ramp and step steer manoeuvres compared to a simpler scheme in which the traction force and yaw moment targets are realised via equal torque contributions from the front and rear wheel actuators. The scheme uses a simple method for determining the four wheel torques and does not require complex online optimisation, and can therefore be practically applied on real electric vehicles.

\section{Acknowledgment}

This research received funding from the European Union Seventh Framework Programme FP7/20072013 under grant agreement no. 284708.

\section{References}

[1] Esmailzadeh E, Goodarzi A, Vossoughi GR. Optimal yaw moment control law for improved vehicle handling. Mechatronics. 2003;13(7):659-675.

[2] Sakai Si, Sado H, Hori Y. Motion control in an electric vehicle with four independently driven in-wheel motors. IEEE/ASME Transactions on Mechatronics. 1999;4(1):9-16.

[3] Gillespie TD. In: Fundamentals of Vehicle Dynamics. SAE; 1992.

[4] Doumiati M. Optimal wheel torque distribution for a four-wheel-drive fully electric vehicle. SAE International Journalk of Passenger Cars. 2013;6(1):128-136. 
[5] Tjønnås J, Johansen TA. Stabilization of automotive vehicles using active steering and adaptive brake control allocation. IEEE Transactions on Control System Technology. 2010;18(3):545-558.

[6] Tavasoli A, Naraghi M. Comparison of static and dynamic control allocation techniques for integrated vehicle control. In: 18th IFAC World Congress. Milano, Italy; 2011.

[7] Chen Y, Wang J. Energy-efficient control allocation with applications on planar motion control of electric ground vehicles. In: American Control Conference (ACC); 2011. p. 2719-2724.

[8] Chen Y, Wang J. Fast and global optimal energy-efficient control allocation with applications to over-actuated electric ground vehicles. IEEE Transactions on Control Systems Technology. 2012;20(5):1202-1211.

[9] Yuan X, Wang J, Colombage K. Torque distribution strategy for a front and rear wheel driven electric vehicle. In: 6th IET International Conference on Power Electronics, Machines and Drives (PEMD 2012). Bristol, United Kingdom; 2012.

[10] Pennycott A, De Novellis L, Gruber P, Sorniotti A. Enhancing the energy efficiency of fully electric vehicles via the minimization of motor power losses. In: IEEE International Conference on Systems, Man, and Cybernetics. Manchester, United Kingdom; 2013.

[11] Härkegård O. Efficient active set algorithms for solving constrained least squares problems in aircraft control allocation. In: Decision and Control, 2002, Proceedings of the 41st IEEE Conference on; 2002. p. 1295-1300.

[12] Härkegård O, Glad ST. Resolving actuator redundancyoptimal control vs. control allocation. Automatica. 2005;41(1):137-144.

[13] Rahman KM, Gopalakrishnan S, Fahimi B, Rajarathnam AV, Ehsani M. Optimized torque control of switched reluctance motor at all operational regimes using neural network. IEEE Transactions on Industry Applications. 2001;37(3):904-913.

[14] Assanis D, Delagrammatikas G, Fellini R, Filipi Z, Liedtke J, Michelena N, et al. Optimization approach to hybrid electric propulsion system design. Journal of Structural Mechanics. $1999 ; 27(4): 393-421$.

[15] Economic Commission for Europe. Uniform Provisions concerning the approval of vehicles of categories M, N and $\mathrm{O}$ with regard to braking. Addendum 12: Regulation No. 13. 2008.

[16] Härkegård O. Backstepping and control allocation with applications to flight control. Department of Electrical Engineering, Linköping University, Sweden; 2003.

[17] Petersen JAM, Bodson M. Constrained quadratic programming techniques for control allocation. Control Systems Technology, IEEE Transactions on. 2006;14(1):91-98. 